\title{
Detection of coherent acoustic oscillations in a quantum electromechanical resonator
}

Cite as: Appl. Phys. Lett. 90, 043101 (2007); https://doi.org/10.1063/1.2432954

Submitted: 20 September 2006 • Accepted: 18 December 2006 • Published Online: 22 January 2007

Florian W. Beil, Robert H. Blick, Achim Wixforth, et al.

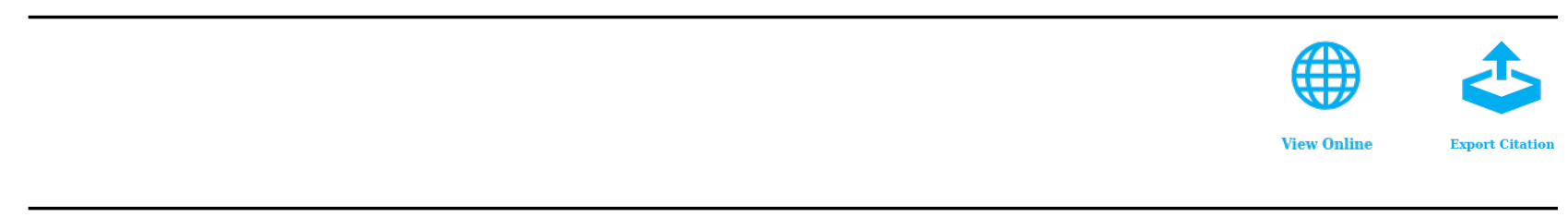

\section{ARTICLES YOU MAY BE INTERESTED IN}

Effects of electron confinement on the acoustoelectric current in suspended quantum point contacts

Applied Physics Letters 110, 223102 (2017); https://doi.org/10.1063/1.4984228

Flip-chip gate-tunable acoustoelectric effect in graphene

Journal of Applied Physics 124, 194302 (2018); https://doi.org/10.1063/1.5047211

Surface acoustic wave propagation in graphene film

Journal of Applied Physics 118, 104901 (2015); https://doi.org/10.1063/1.4930050

母QBLOX

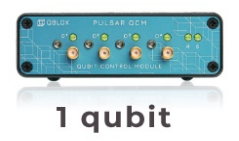

Shorten Setup Time Auto-Calibration More Qubits

Fully-integrated Quantum Control Stacks Ultrastable DC to $18.5 \mathrm{GHz}$ Synchronized $<<1$ ns Ultralow noise

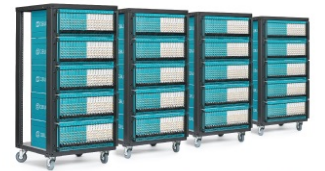

100s qubits

visit our website > 


\title{
Detection of coherent acoustic oscillations in a quantum electromechanical resonator
}

\author{
Florian W. Beil \\ Center for NanoScience and Sektion Physik, Ludwigs-Maximilians-Universität, Geschwister-Scholl-Platz, 1, \\ 80539 München, Germany \\ Robert H. Blick ${ }^{\text {a) }}$ \\ Department of Electrical and Computer Engineering, University of Wisconsin-Madison, \\ 1415 Engineering Drive, Madison, Wisconsin 53706 \\ Achim Wixforth \\ Lehrstuhl für Experimentalphysik I, Universität Augsburg, Universitätsstraße 1, 86159 Augsburg, Germany \\ Werner Wegscheider and Dieter Schuh \\ Institut für Angewandte und Experimentelle Physik, Universität Regensburg, 93040 Regensburg, Germany \\ Max Bichler \\ Walter Schottky Institut, Am Coloumbwall 3, 85748 Garching, Germany
}

(Received 20 September 2006; accepted 18 December 2006; published online 22 January 2007)

Coherent control of occupation numbers in quantum mechanical multilevel systems is widely studied driven by its application in lasers and its prospects for quantum computational elements. Here the authors present a nanoelectromechanical resonator equivalent to the coherent control of a quantum mechanical two level system. The distinct eigenmodes of a nanomechanical beam resonator represent the two levels whose amplitude mode occupation numbers are controlled by a frequency matched acoustic excitation, mediated by a pulsed surface acoustic wave. They show that similar to quantum mechanical systems it is possible to transfer occupation numbers from one mode to another by matched acoustic pulses. () 2007 American Institute of Physics. [DOI: $10.1063 / 1.2432954]$

A straightforward method to control occupation numbers in quantum mechanical two level systems is to apply short pulses of light, which are frequency matched to the transition frequency between the two levels. ${ }^{1}$ The transfer of occupation number between two levels, usually termed as Rabi oscillations, relies on a system's state which can be described as a superposition of distinct modes, attributed eigenstates, being coupled by an external perturbation of the system. This pumping of atoms into an excited state results in an occupation inversion, which is the precondition for lasing operation.

Consequently, a mechanical analog can be realized once eigenstates are identified and appropriately addressed. For quantum electromechanical (QEM) systems, such as nanomechanical resonators, the different eigenstates correspond to different vibrational modes. Any displacement of the resonator can be described as a superposition of eigenmodes, whereas each eigenmode $j$ contributes with an amplitude or, in analogy to the quantum mechanical counterpart, mode occupation number $A_{j}$ to the overall motion. Usually, it is not possible to directly control the mode occupation numbers $A_{j}$, since thermal noise or nonlinear coupling determines the mode distribution. In the macroscopic extreme classical coupling of modes can occur for suspension bridges due to pulsed wind excitation, as the Takoma bridge disaster has shown ${ }^{2}$ or due to pedestrians crossing the Millenium Bridge in London. ${ }^{3}$

In the experiment described here a surface acoustic wave (SAW) of Rayleigh type, ${ }^{4}$ properly described as a nanoquake, on GaAs is frequency matched to the transition fre-

\footnotetext{
a) Author to whom correspondence should be addressed; electronic mail: blick@engr.wisc.edu
}

quency between two eigenmodes of a nanomechanical beam resonator (see Fig. 1). ${ }^{5}$ In full equivalence to atoms pumped by light, the resonator represents the multilevel system, whereas the phonons excited by the acoustic wave correspond to the photons. The amplitude of the resonator beam's first eigenmode shows similar behavior as observed for occupation numbers in the quantum mechanical equivalent, namely, Rabi-like oscillations versus pump power and a Rabi-like splitting. ${ }^{6}$

To demonstrate the mechanical equivalence to pumped atoms, the suspended, gold covered nanobeam is placed in the line of fire of the interdigitated transducer (IDT) generating the SAW. The comblike IDT structure allows generation of SAWs by the inverse piezoelectric effect, which converts an applied radio frequency (rf) signal to the electrodes into mechanical stress, as the electric potential drops between each finger pair. If the frequency $f_{\text {saw }}$ of the signal meets the resonance condition $f_{\text {saw }}=v_{\text {saw }} / \lambda$, a coherent acoustic sound wave is generated. The IDT, however, provides a certain bandwidth, being determined by the number of finger pairs, hence allowing for a moderate frequency tuning, as employed in Fig. 3, for example, Ref. 5. The frequency is defined by the sound velocity $v_{\text {saw }}=2865 \mathrm{~m} \mathrm{~s}^{-1}$ and the lithographically defined SAW wavelength $\lambda$ (corresponding to the pitch between the SAW electrodes). As the length of the beam $L$ is matched to $\lambda / 2$, the two clamping points move in counterphase and the beam is periodically stressed by this motion.

The setup is placed in a strong magnetic field, which allows magnetomotive excitation and detection of the beam's off-plane eigenmode. ${ }^{7}$ Driving an alternating current at frequency $f$ with power $P_{\text {res }}$ along the conduction top metallic 




FIG. 1. (Color online) Top and lower right show setup of the experiment. The suspended beam is placed in the line of fire of a transducer which generates a surface acoustic wave (SAW) of frequency $f_{\text {saw. }}$. The SAW displaces the anchoring points of the resonator beam in counterphase and periodically stresses the beam. The inset right of the center shows an actual SEM micrograph of the resonator beam and the clamping points. An inplane magnetic field $\boldsymbol{B}$ allows detection of the beam's off-plane eigenmode by impedance spectroscopy. Lower left: schematic representation of the SAW induced pumping from the first to the third eigenmode of the beam $\omega_{3}-\omega_{1}=\Omega_{\text {saw }}$.

layer causes a Lorentz force, which when in resonance excites the beam's mechanical modes with an amplitude $A$. In Fig. 2(a) we traced the power dependence of this amplitude with $A=A_{\text {res }}$. The induced motion changes the beam's acoustoelectrical impedance $Z_{\text {beam }}$, which we measure as an effective amplitude of the oscillations $A=A_{\text {osc }}$ in Fig. 2(b). In turn it exhibits a mechanical resonance in the reflected power. Measuring the scattering parameter $S_{11} \sim\left(1-A_{\text {osc }}\right)$ versus driving frequency $f$ reveals the fundamental mechanical resonance, shown in Fig. 2.

For large amplitudes $A \sim A_{\text {res }}$, nonlinear effects come into play and distribute energy among the beam's eigenmodes. This is shown in Fig. 2(a), where we plot $A_{\text {res }}$ against the probe power $P_{\text {res }}$ applied at the beam. Above $P=$ $-56 \mathrm{dBm}$ nonlinear effects kick in and higher eigenmodes are excited $\left(A_{\text {res }} \sim A_{1}+A_{3}\right)$. The necessary adjustment of the SAW frequency $\Omega_{\text {saw }}=2 \pi f_{\text {saw }}$ to the transition frequency of the beam's first eigenmode to its third harmonic $f_{\text {trans }}=f_{3}$ $-f_{1} \sim \omega_{3}-\omega_{1}=\Omega_{\text {saw }}$ was calculated by finite element simulations, achieving sufficient accuracy for the IDT's bandwidth (Fig. 1).

For pumping the transition from the first to the third eigenmode, short acoustic pulses were applied. The minimal pulse length is determined by the pitch of the IDT and is of the order of 100 ns. In Fig. 2(b) $A=A_{\text {osc }} \sim A_{1}$ exhibits oscillations against increased SAW pulse amplitude $A_{\text {saw }}$. This effect is best visualized when coding the reflected signal from the beam in a grayscale plot [Fig. 2(c)]. These oscillations of $S_{11} \sim\left(1-A_{\text {osc }}\right)$ vs $A_{\text {saw }}$ are equivalent to oscillations of occupation numbers in a two level system usually plotted versus pump power; hence we term these oscillations as acoustical Rabi-like oscillations (AROs). Increasing the magnetoimpedance probe power $P_{\text {res }}$, the AROs disappear when increasing the power above the limit of nonlinear response at $-56 \mathrm{dBm}$ [Fig. 2(b)]. $P_{\text {res }}$ corresponds to the power of the network analyzer applied to the sample.
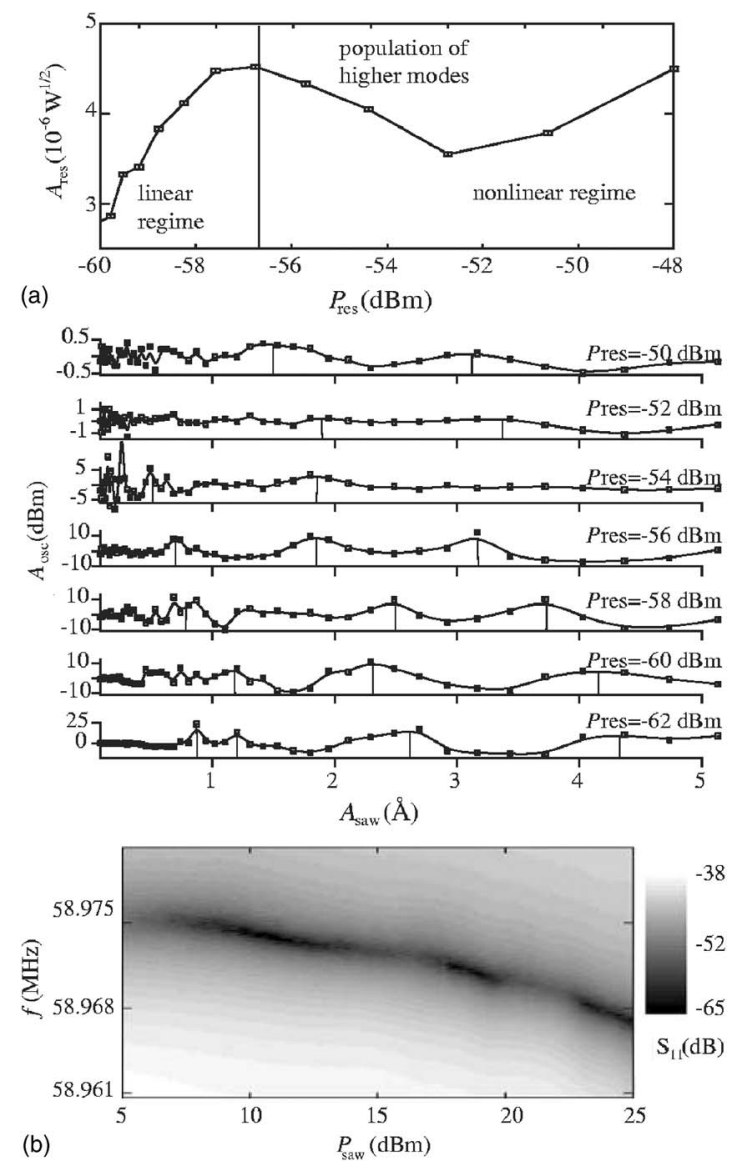

FIG. 2. (a) Dependence of the resonator oscillation amplitude $A=A_{\text {res }}$ on the applied magneto impedance probe power $P_{\text {res }}$. Above $-56 \mathrm{dBm}$ the nonlinear coupling to higher modes leads to a deviation from the linear increase. (b) Upper part: amplitudes $A=A_{\text {osc }}$ of acoustic Rabi-like oscillations (ARO) for different probe powers $P_{\text {res }}$. The AROs start to vanish for probe powers above the nonlinear limit of $-56 \mathrm{dBm}$. Lower part: visualization of the ARO in the reflectance $S_{11} \sim\left(1-A_{\text {osc }}\right)$ at the beam vs acoustic pulse power $P_{\text {saw. }}$. In this plot an increased modal occupation $A \sim A_{1}$ corresponds to darker areas, whereas the lighter regions correspond to low $A_{1}$, due to acoustic coupling to a higher eigenmode $A_{3}$.

Another characteristic feature which the quantum electromechanical resonator reveals is the characteristic dependence of the ARO on pump frequency $f_{\text {saw }}$ as expected from quantum mechanical systems. In Fig. 3(a) the measured amplitude $A \sim A_{1}$ is plotted against a broad range of SAW frequencies. This shows that the observed mode coupling only occurs for a specific pulse center frequency $F_{\text {saw }}=f_{\text {trans }}$. Comparing the measured curve to the Fourier transform of the applied SAW signal $A_{\mathrm{FT}}$ at $f_{\text {trans }}$ allows us to extract the transition frequency of the first to the third eigenmode to be $302.9 \mathrm{MHz}$, which lies in the active frequency range of the IDT. Here, we are able to cover a broader frequency range, since we operate the IDTs in pulsed mode. Hence, we find evenly spaced harmonics in the range of 280-340 MHz. The shape of $A$ vs $f_{\text {saw }}$ around the transition frequency for two different SAW powers shows the form of two separated resonances [Fig. 3(b)]. This again is expected for the coupling of two states leading to Rabi-like splitting of the energy levels.

To model acoustic pumping of mode transitions we adopt the theory developed for quantum mechanical systems to the acoustic case. The amplitude of the beam resonator's mechanical amplitude $A$ in the linear regime is described as a superposition of eigenmodes $A_{j}$, whereas each mode $j$ contributes to it with specific wave function shapes $\psi_{1}$ and $\psi_{3}$. 

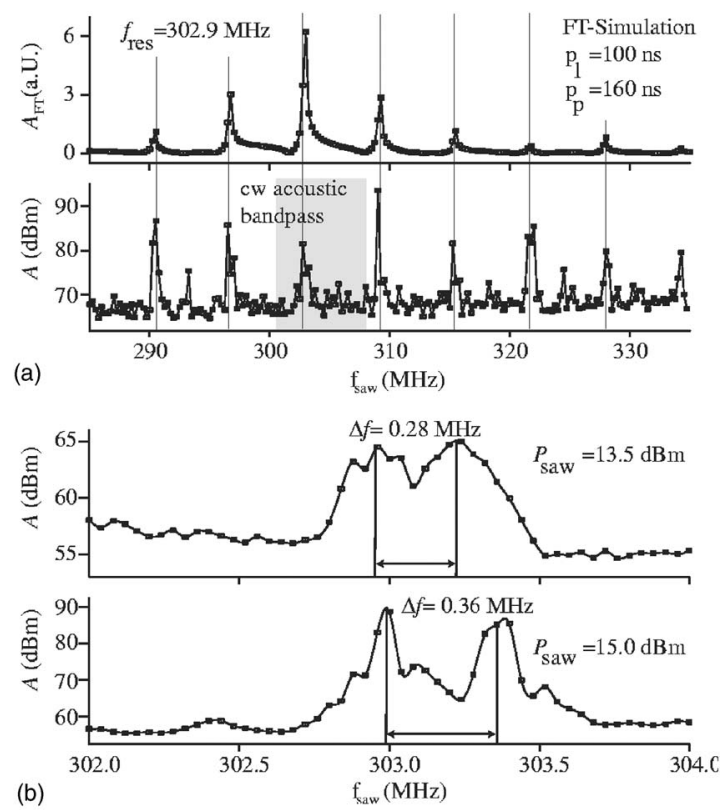

(b)

$\mathrm{f}_{\mathrm{saw}}(\mathrm{MHz})$

FIG. 3. (a) ARO vs SAW frequency $f_{\text {saw }}$ and calculated component of the applied acoustic signal's Fourier transform $\left(A_{\mathrm{FT}}\right)$ at $f=f_{\text {trans }}$. From this $f_{\text {trans }}$ is evaluated to $f_{\text {trans }}=303.2 \mathrm{MHz}$. (b) Measured amplitude $A$ for $f_{\text {saw }}$ close to $f_{\text {trans }}$ for two different SAW pulse powers. The acoustic frequency splitting decreases for lower $P_{\text {saw }}$

The SAW induced periodic stress acting on the beam is included in the Euler-Bernoulli beam equations by an additional term, ${ }^{8,9}$ which will make the $A_{j}$ become time dependent. Using the orthogonality of eigenmodes ${ }^{10}$ in the rotating wave approximation, ${ }^{5}$ which assumes that contributions from eigenmodes with frequencies very different from $f_{1}+f_{\text {trans }}$ can be neglected, we obtain the following coupled differential equation for the acoustically coupled eigenmodes $A_{1}$ and $A_{3}$ :

$$
\begin{aligned}
& \frac{\partial^{2} A_{1}}{\partial t^{2}}+2 i \omega_{1} \frac{\partial A_{1}}{\partial t}=-\Omega_{13}^{2} A_{3}, \\
& \frac{\partial^{2} A_{3}}{\partial t^{2}}+2 i \omega_{3} \frac{\partial A_{3}}{\partial t}=-\Omega_{13}^{2} A_{1} .
\end{aligned}
$$

Here, $\omega_{j}=2 \pi f_{j}$, and $\Omega_{13}$ is the acoustic Rabi-like frequency, which is determined by the mass per unit length $\mu$ of the beam, the mismatch between the SAW frequency and the transition frequency $\Delta=f_{\text {trans }}-f_{\text {saw }}$.

$$
\Omega_{13}=\int_{0}^{L} \psi_{1} \frac{\partial^{2}}{\partial x^{2}} \psi_{3} d x \frac{F_{\text {saw }}}{\mu} e^{i \Delta t} .
$$

This integral depends on the mode shapes $\psi_{1}$ and $\psi_{3}$ of the two coupled modes integrated over the length $L$ of the beam and the force $F_{\text {saw }}$ the SAW exerts on the beam. The force can be calculated from simple beam theory with the assumption of elliptic motion of the suspension points with amplitude $A_{\text {saw }}$.

The two coupled modes will periodically exchange energy, whereas the acoustic Rabi frequency determines the period of this exchange. The amplitude of the SAW can be estimated by the acoustic power injected by the IDT, leading to typical frequencies of $\Omega_{13}$ in the megahertz regime. This corresponds to the observed Rabi-like splitting in Fig. 3, which allows direct evaluation of $\Omega_{13}$. Solving the coupled differential equation [Eq. (1)] leads to the theoretical curves
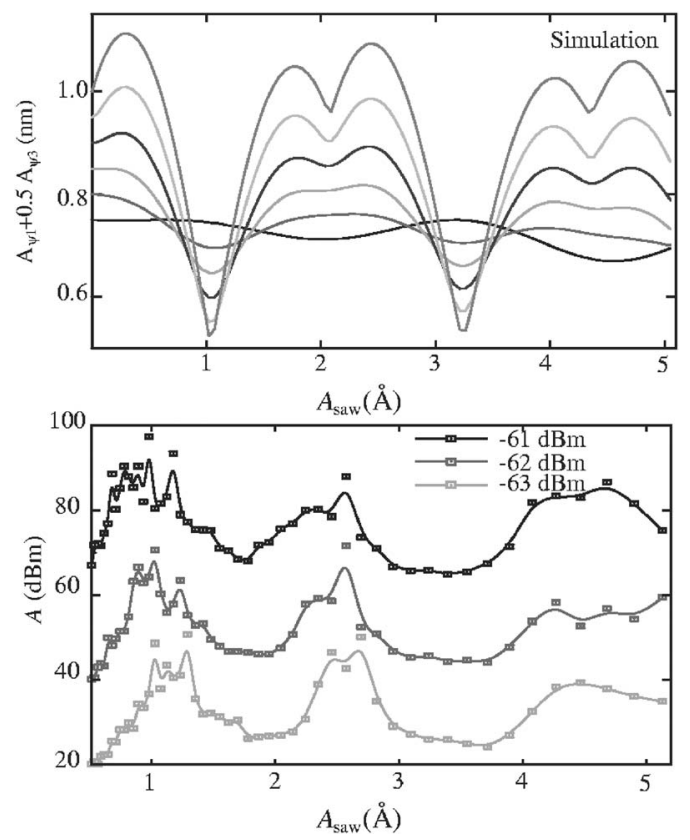

FIG. 4. Simulated (top) and measured (bottom) AROs in amplitude A. To model a contribution from the third eigenmode to the reflected signal to the first one, a superposition of $A \sim A_{1}+0.5 A_{3}$ was calculated for different SAW pulse amplitudes. The simulation shows vanishing ARO for increased occupation of $A_{3}$ compared to $A_{1}$, as observed in Fig. 2(a).

shown in Fig. 4. For the different traces the parameters $A_{1}$ and $A_{3}$ are varied in combination as $A_{1}+0.5 A_{3}$, ensuring that the fundamental mode at $A_{1}$ and the higher harmonic at $A_{3}$ are excited. The calculations resemble the experimentally observed ARO in Fig. 4 for the range of applied SAW amplitudes.

In summary, we demonstrated that in analogy to the control of occupation numbers in a quantum mechanical two level system, the mode occupation numbers in quantum electromechanical resonators can be controlled by pulsed acoustic excitation. Hence, it is possible to coherently control the mode occupation numbers in a nanomechanical resonator, which can be exploited for achieving occupation inversion necessary for a mechanical equivalent to lasers, termed the "phaser."

This work was supported by the Deutsche Forschungsgemeinschaft (B1-487/3) and the Air Force RSO (F49620-031-0420).

${ }^{1}$ A. Zrenner, E. Beham, S. Stufler, F. Findeis, M. Bichler, and G. Abstreiter, Nature (London) 418, 612 (2002).

${ }^{2}$ K. Y. Billak and R. H. Scanlan, Am. J. Phys. 59, 118 (1991).

${ }^{3}$ S. H. Strogatz, D. M. Abrams, A. McRobie, and B. Eckhardt, Nature (London) 483, 43 (2005).

${ }^{4}$ L. D. Landau and E. M. Lifhitz, Theory of Elasticity (ButterworthHeinemann, Oxford, 1999), Vol. 1, p. 97.

${ }^{5}$ A. Wixforth, J. Scriba, M. Wassermeier, J. P. Kotthaus, G. Weimann, and W. Schlapp, Phys. Rev. B 40, 7874 (1989).

${ }^{6}$ I. I. Rabi, N. F. Ramsey, and J. Schwinger, Rev. Mod. Phys. 26, 167 (1954).

${ }^{7}$ R. H. Blick, A. Erbe, L. Pescini, A. Kraus, D. V. Scheible, F. W. Beil, E. M. Höhberger, A. Hoerner, J. Kirschbaum, H. Lorenz, and J. P. Kotthaus, J. Phys.: Condens. Matter 14, R905 (2002).

${ }^{8}$ K. Magnus and K. Popp, Schwingungen (Teubner, Stuttgart, 1997), Vol. 1, p. 15.

${ }^{9}$ K. F. Graff, Wave Motion in Anelastic Solids (Ohio State University Press, Columbus, 1975), Vol. 1, p. 42.

${ }^{10}$ C. Cohen-Tannoudji, Quantum Mechanics (Wiley, New York, 1996), Vol. 2, p. 137. 